\title{
Dialkylcarbamoyl chloride-impregnated dressing for the prevention of surgical site infection in women undergoing cesarean section: a pilot study
}

Paweł J. Stanirowski, Anna Kociszewska, Krzysztof Cendrowski, Włodzimierz Sawicki

Department of Obstetrics, Gynecology and Oncology, Second Faculty of Medicine, Medical University of Warsaw, Mazovian Bródno Hospital, Warsaw, Poland

Submitted: 28 August 2014

Accepted: 22 November 2014

Arch Med Sci 2016; 12, 5: 1036-1042

DOI: $10.5114 /$ aoms.2015.47654

Copyright @ 2015 Termedia \& Banach

\section{Abstract}

Introduction: Incisional surgical site infections (SSIs) occur in approximately $1.8-9.2 \%$ of patients undergoing cesarean section (CS) and contribute to prolonged hospitalization time and increased treatment costs. Dressings impregnated with dialkylcarbamoyl chloride (DACC) are an innovative approach to wound treatment based on a solely physical mechanism of action, and therefore can be used safely and without time restrictions in women during the puerperal and lactation period.

Material and methods: A single-blinded randomized, controlled pilot study was conducted at the Mazovian Bródno Hospital, a tertiary care hospital, between December 2013 and March 2014, and it evaluated the presence of superficial and deep SSIs in patients during the first 14 days after a CS. Patients were randomly allocated to receive treatment with either a DACC dressing or a standard surgical dressing.

Results: One hundred and forty-two patients after planned or emergency CS were enrolled in the study. No significant differences between the groups were observed with regard to patients' basic demographic and perioperative characteristics. The rate of superficial and deep SSIs was $2.8 \%$ in the group of patients who received a DACC dressing compared to $9.8 \%$ in the group with a standard surgical dressing $(p=0.08)$. Patients with SSIs who received a standard surgical dressing required systemic antibiotic therapy significantly more frequently $(p=0.03)$. Based on the logistic regression model developed, the pre-pregnancy body mass index was the only statistically significant risk factor for SSI $(p=0.015)$.

Conclusions: The results of the pilot study indicate a decreasing tendency of the SSI rate in patients after a CS who received DACC impregnated dressings.

Key words: dialkylcarbamoyl chloride, surgical site infection, cesarean section.

\section{Introduction}

A rapid increase in the rate of cesarean sections (CS) observed within the last 30 years, especially in developed countries, has resulted in the fact that currently it is one of the most frequently performed surgical procedures. According to the literature data and depending on the region of the world, $0.4-40.5 \%$ of all deliveries have a surgical outcome [1]. Incisional wound infection defined according to the Centers for Disease Control and Prevention (CDC) criteria as a superficial or deep surgical site

\author{
Corresponding author: \\ Paweł J. Stanirowski MD \\ Department of Obstetrics, \\ Gynecology and Oncology \\ Second Faculty of Medicine \\ Medical University of Warsaw \\ Mazovian Bródno Hospital \\ 8 Kondratowicza St \\ 03-242 Warsaw, Poland \\ Phone: +48 223264058 \\ E-mail: stanirowski@gmail. \\ com
}


infection occurs in approximately $1.8-9.2 \%$ of patients after a CS, being one of the most common complications that may lead to wound dehiscence or systemic infections in case of lack of sufficient prophylaxis or inappropriate treatment [2-8]. Increased morbidity, prolonged hospitalization time, increased rate of hospital readmissions and growing treatment costs are all consequences of the above [9].

Dressings impregnated with dialkylcarbamoyl chloride (DACC) are an innovative approach used in the treatment of venous, arterial, diabetic and pressure ulcers, burns, post-traumatic and post-operative wounds [10-13]. Dialkylcarbamoyl chloride, a hydrophobic derivative of fatty acids, irreversibly binds microorganisms as a result of an interaction between hydrophobic particles in the presence of the aqueous medium, therefore allowing for elimination of bacteria and fungi during dressing replacement. The mechanism of action is based on the fact that the majority of pathogens isolated from infected wounds express high to moderate cell surface hydrophobicity (CSH), which on the one hand enables cell attachment and infection initiation by microbes, but on the other hand facilitates their aggregation on the surface of a hydrophobic dressing under moist wound conditions [14-17]. As a result, the number of pathogenic microorganisms in the wound bed is significantly reduced, and their proliferation as well as toxin production is limited $[18,19]$. Additionally, the results of recently published in vitro studies demonstrated a separate mechanism of action present within hydrophobic dressings, responsible for accelerated wound healing, namely stimulation of fibroblast proliferation and migration [20]. Due to the fact that dressings have a solely physical mechanism of action that is not accompanied by a release of additional antimicrobial substances, the risk of cytotoxicity and sensitization has been eliminated and there are no risks associated with resistance development among bacteria and fungi responsible for wound infections. As a result, hydrophobic dressings can be used safely and without time restrictions, including in women during the puerperal and lactation period.

Taking into account the continuously increasing number of CS and a wide spread of factors responsible for wound healing disturbances in the population of women of reproductive age, such as obesity, diabetes and nicotinism, it is necessary to search for new, more effective methods to prevent infections of surgical wounds.

This study aimed to assess the efficacy of dressings impregnated with DACC in the prevention of incisional surgical site infections in patients undergoing cesarean section.

\section{Material and methods}

A single-blinded randomized, controlled pilot study was conducted at the Department of Obstetrics, Gynecology and Oncology, Mazovian Bródno Hospital, Warsaw, Poland between December 2013 and March 2014. Mazovian Bródno Hospital is a tertiary care and academic hospital for the Medical University of Warsaw, performing approximately 1300 deliveries per year, with the CS rate of $51.8 \%$ in the study period. Expedited approval for this study was obtained from the Ethics Committee at the Medical University of Warsaw, and written informed consent was gained from all participants. Patients after a planned or emergency CS were enrolled in the study. The inclusion criteria were as follows: age above 18 years, single or multiple pregnancy, mental and physical capability to provide written consent for participation in a clinical trial, and patient's declared compliance with the study plan. Women less than 18 years old or unable to give informed consent were excluded from the study. Patients were randomized into 2 groups depending on the dressing that had been applied. Patients in whom after a cesarean section a dressing impregnated with DACC (Sorbact Surgical Dressing, ABIGO Medical $A B$, Sweden) was used constituted the study group, whereas patients who received a standard surgical dressing were included in the control group. Simple randomization with the $1: 1$ allocation ratio performed by an operating theater nurse was used to alternate patients enrolled for alternate dressings - even number: DACC-impregnated dressing; odd number: standard surgical dressing.

Data on patient characteristics, pre-, peri- and postoperative course were taken from hospital medical records. The analysis included age, race, parity, pre-pregnancy weight, weight gain during pregnancy, pre-pregnancy body mass index (BMI), gestational age, history of pre-gestational or gestational diabetes mellitus, chronic or pregnancy-induced hypertension, previous CS, tobacco use during pregnancy, mode of CS (planned/ emergency), duration of surgery, surgeon experience, hemoglobin levels prior to and $24 \mathrm{~h}$ after surgery, and hospitalization time after surgery. An emergency CS was defined based on the time between a decision and skin incision below $30 \mathrm{~min}$. The surgery time was measured between skin incision and skin closure. The hospitalization time after surgery was the number of days from the CS (day 0) to the patient's discharge from hospital. The surgeon's experience was determined based on the medical position and work experience, and classification to one of three groups was performed: resident - a medical doctor during specialization in obstetrics, an obstetrics specialist working for 5 years at most, or an obstetrics specialist working for more than 5 years. 
In all patients enrolled in the study a transverse skin incision (Pfannenstiel) followed by a transverse uterine incision in its lower segment was applied. For fascial, subcutaneous tissue and skin incision closure, continuous antibacterial braided absorbable suture (Vicryl Plus 1, Ethicon, USA), single monofilament absorbable suture (Monosyn 2/0, B. Braun Melsungen AG, Germany) and subcuticular continuous monofilament non-absorbable suture (Prolene 2-0, Ethicon, USA), respectively, were used. All participants received an intravenous antibiotic dose as prophylaxis $1 \mathrm{~g}$ of cefazolin 0-30 min prior to the start of surgery. Additionally, in all patients wound irrigation with an octenidine solution (Octenisept, Schülke \& Mayr $\mathrm{GmbH}$, Germany) was performed prior to the closure of subcutaneous tissue. The surgical team was blinded to the dressing type until skin closure.

Pursuant to the valid study protocol, in all subjects dressings remained in situ for the first $48 \mathrm{~h}$ after surgery unless there were reasons for dressing replacement such as excessive wound bleeding and/or dressing detachment. After $48 \mathrm{~h}$ dressings were removed in both groups, and the first clinical wound evaluation was performed. According to the management strategy at the clinic, patients were discharged on the third day after surgery and were recommended to re-visit on day 7 in order to have skin suture removed. The second clinical wound assessment was performed at this visit. The third and final wound evaluation was scheduled on day 14 after a CS. On the discharge day all patients for whom less than 14 days had passed since surgery received detailed instructions regarding surgical site infections (SSIs) and were informed about the need to report at hospital in case at least one of the following symptoms was observed: fever, suppurative secretion from the surgical site, redness, edema, warmth, pain or tenderness of the surgical site area. Patients who did not report for follow-up visits 7 and 14 days after a CS were excluded from the final analysis.

At each surgical wound evaluation scheduled in the study, symptoms of superficial or deep SSI were analyzed according to the CDC criteria [2]. Additionally, the presence of surgical wound dehiscence, defined as separation of the skin, subcutaneous tissue and/or fascia resulting from an incisional SSI, was evaluated. Other parameters subject to the analysis in the group of patients with wound infection were as follows: time of wound infection occurrence (defined as the period from day 0 to the day when first infection symptoms developed), need for systemic antibiotic treatment, need for hospital readmission and need for a surgical intervention. All surgical wound evaluations during patient hospital stay, follow-up visits and self-referral to hospital, as well as SSI classification according to the CDC criteria, were performed by one of the authors (PS).

From all patients with clinical symptoms of SSI, wound swabs were collected in order to perform a microbiological analysis and to identify the responsible pathogen (Copan Sterile Transport Swab, Copan Diagnostics, USA).

CONSORT 2010 guidelines for randomized controlled trials including a checklist and flow diagram were applied to ensure the quality of the conducted study [21].

\section{Statistical analysis}

The primary outcome in this study was development of superficial or deep SSI within the first 14 days after a CS. In order to assess whether individual pre-, peri- and post-operative variables affect the development of surgical site infection in women undergoing CS, a logistic regression model with forward selection was designed. The model included the following variables: age, race, parity, gestational age, pre-pregnancy weight, weight gain during pregnancy, pre-pregnancy BMI, pre-gestational or gestational diabetes mellitus, chronic or pregnancy-induced hypertension, previous CS, tobacco use during pregnancy, mode of CS, duration of surgery, surgeon experience, hemoglobin levels prior to and $24 \mathrm{~h}$ after surgery, hospitalization time after surgery as well as dressing type.

A statistical analysis was performed using SPSS 17.0 for Windows software (SPSS Inc, Chicago, IL). The Shapiro-Wilk test was used for a normality analysis. Continuous variables were compared by the $t$ test, or the Mann-Whitney $U$ test, as appropriate. For categorical variables the $\chi^{2}$ test or the Fisher exact test were applied. Statistical significance was set at $p<0.05$.

\section{Results}

In the period between December 2013 and March 2014 at the Department of Obstetrics, Gynecology and Oncology 193 patients underwent CS, and 31 of them did not meet the study inclusion criteria. One hundred and sixty-two participants were randomized, however 20 women (10 in each group) did not report for scheduled follow-up visits and were excluded from the analysis, leaving a total of 142 eligible patients. The number of participants evaluable for final analysis was equal in both groups - 71 patients in the group with a DACC-impregnated dressing (study group) and in the group with a standard surgical dressing (control group). The patient recruitment process and randomization are summarized in the CONSORT 2010 flow diagram (Figure 1).

Eighteen $(25.3 \%)$ patients in the study group and $17(23.9 \%)$ patients in the control required 


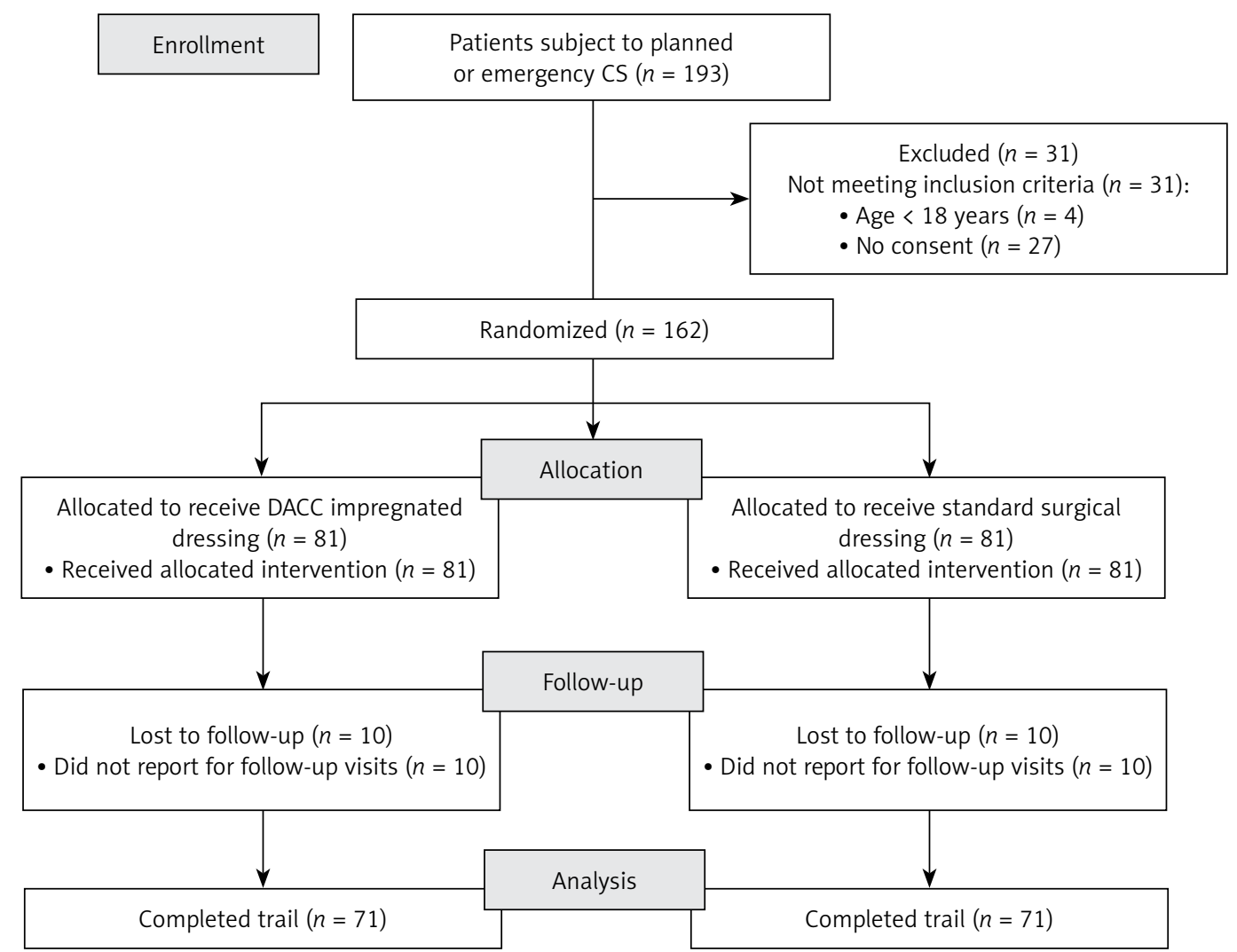

Figure 1. CONSORT 2010 flow diagram of the recruitment process and randomization

dressing replacement within the first $48 \mathrm{~h}$ following surgery as a result of wound hemorrhage $(p=0.84)$. There were no cases of dressing detachment in both groups.

Demographic, peri- and postoperative characteristics are shown in Table I. There were no significant differences between the two groups.

Primary and secondary study outcomes are summarized in Table II. The rate of surgical site infections in the group with a DACC-impregnated dressing was $2.8 \%$ (2 cases of superficial SSI), whereas in the group with a standard surgical dressing it was $9.8 \%$ (6 cases of superficial and 1 case of deep SSI) $(p=0.08)$. One $(1.4 \%)$ patient in the control group developed surgical wound dehiscence due to infection. The same patient required rehospitalization and a surgical intervention due to necrotizing fasciitis. In the study group there were no cases of wound dehiscence, and none of the patients who received a DACC-impregnated dressing required hospital readmission and/or surgical intervention. In 5 (7.0\%) cases of wound infection in the control group systemic antibiotic treatment was instituted, whereas it was not necessary in the study group $(p=0.03)$. The mean time to first symptoms of wound infection was 10.5 and 8.8 days in the study and control group, respectively $(p=0.24)$.
The microbiological analysis of cultures from infected wounds demonstrated Staphylococcus epidermidis in 2 and 3 cases of SSIs in the study and control group, respectively. In the remaining patients in the control group wound infection was caused by Pseudomonas aeruginosa (2x), Streptococcus sp. (1×) and methicillin-resistant S. epidermidis (1x).

The logistic regression model used to determine the effects of selected parameters on SSI development was statistically significant $\left(\chi^{2}(1)=5.52\right.$; $p=0.019)$ and explained $10.1 \%$ of observed variance for the dependent variable $\left(R^{2}\right.$ Nagelkerke $=$ 0.101). The Hosmer-Lemeshow test demonstrated that the model is appropriate for data collected: $\chi^{2}(8)=6.13 ; p=0.632$. According to the logistic regression analysis, only the pre-pregnancy $\mathrm{BMI}$ was a significant predictor of wound infection $(B=0.13 ; W=5.96 ; p=0.015 ; \exp (B)=1.139$; 95\% Cl: 1.026-1.265).

\section{Discussion}

This study aimed to assess the efficacy of dressings impregnated with DACC in the prevention of wound infection in patients after a CS. To our knowledge there have not been any studies published to date on the use of such dressings in the prevention of surgical wound infection. The re- 
Table I. Demographic, peri- and postoperative characteristics

\begin{tabular}{|c|c|c|c|}
\hline Parameter & Study group $(n=71)$ & Control group $(n=71)$ & $P$-value \\
\hline Age [years] & $30.9 \pm 4.5(19-41)$ & $31.2 \pm 5.1(19-43)$ & 0.64 \\
\hline Race: & & & $>0.999$ \\
\hline Caucasian & $70(98.6)$ & $71(100)$ & \\
\hline Non-Caucasian & $1(1.4)$ & $0(0)$ & \\
\hline Pre-pregnancy weight [kg] & $67.3 \pm 12.4(48-116)$ & $68.8 \pm 16.9(48-116)$ & 0.78 \\
\hline Weight gained during pregnancy [kg] & $14.4 \pm 5.6(0-27)$ & $13.3 \pm 5.6(3-30)$ & 0.26 \\
\hline Pre-pregnancy BMI [kg/m²]: & $24.3 \pm 4.1$ & $25.3 \pm 6.0$ & 0.77 \\
\hline$<25$ & $44(62.0)$ & $41(57.8)$ & 0.61 \\
\hline$\geq 25$ and $<30$ & $18(25.3)$ & $17(23.9)$ & 0.84 \\
\hline$\geq 30$ and $<40$ & $9(12.7)$ & $10(14.1)$ & $>0.999$ \\
\hline$\geq 40$ & $0(0)$ & $3(4.2)$ & 0.24 \\
\hline \multicolumn{4}{|l|}{ Parity: } \\
\hline Nulliparous & $24(33.8)$ & $34(47.9)$ & 0.09 \\
\hline Gestational age [weeks] & $37.9 \pm 2.7(24-41)$ & $38.2 \pm 2.2(28-41)$ & 0.45 \\
\hline Diabetes mellitus: & $7(9.8)$ & $7(9.8)$ & $>0.999$ \\
\hline PGDM & $2(2.8)$ & $4(5.6)$ & 0.68 \\
\hline GDM & $5(7.0)$ & $3(4.2)$ & 0.72 \\
\hline Hypertension: & $7(9.8)$ & $7(9.8)$ & $>0.999$ \\
\hline Pre-pregnancy HTN & $1(1.4)$ & $3(4.2)$ & 0.62 \\
\hline $\mathrm{PIH}$ & $6(8.4)$ & $4(5.6)$ & 0.74 \\
\hline Tobacco use during pregnancy & $4(5.6)$ & $5(7.0)$ & $>0.999$ \\
\hline \multicolumn{4}{|l|}{ Mode of CS: } \\
\hline Planned & $53(74.7)$ & $51(71.8)$ & 0.70 \\
\hline Emergency & $18(25.3)$ & $20(28.2)$ & 0.70 \\
\hline Previous CS & $19(26.8)$ & $17(23.9)$ & 0.70 \\
\hline Duration of surgery [min] & $37.2 \pm 8.0(21-57)$ & $35.3 \pm 12.7(17-105)$ & 0.06 \\
\hline \multicolumn{4}{|l|}{ Surgeon experience: } \\
\hline Resident & $32(45.1)$ & $29(40.9)$ & 0.61 \\
\hline Specialist $\leq 5$ years & $16(22.5)$ & $16(22.5)$ & $>0.999$ \\
\hline Specialist $>5$ years & $23(32.4)$ & $26(36.6)$ & 0.60 \\
\hline Pre-operative hemoglobin [g/dl] & $12.1 \pm 0.9(9.8-14.1)$ & $12.0 \pm 1.0(9.4-14.0)$ & 0.68 \\
\hline Post-operative hemoglobin [g/dl] & $10.7 \pm 1.2(7.6-13.5)$ & $10.7 \pm 1.1(8.0-12.7)$ & 0.64 \\
\hline $\begin{array}{l}\text { Median (range) time of post-operative hospital } \\
\text { stay [days] }\end{array}$ & $4.4 \pm 2.3(3-15)$ & $4.5 \pm 2.5(3-17)$ & 0.59 \\
\hline
\end{tabular}
GDM - gestational diabetes mellitus, HTN - hypertension, PIH - pregnancy-induced hypertension, CS - cesarean section.

sults of the pilot study indicated a decreasing tendency of the observed surgical site infection rate in patients who received DACC dressings. However, when the results obtained were compared to a standard surgical dressing, statistical signif- icance was not achieved, and in order to draw final conclusions it is necessary to perform studies in a larger group of patients. From the point of view of health-care system economics and cost reduction, in the study group there were no 
Table II. Primary and secondary outcomes

\begin{tabular}{|lccc|}
\hline Parameter & Study group $(n=71)$ & Control group $(n=71)$ & $P$-value \\
\hline No. of patients with SSI (\%) & $2(2.8)$ & $7(9.8)$ & $1(1.4)$ \\
\hline No. of patients with SSI and wound dehiscence (\%) & $0(0)$ & $5(7.0)$ \\
\hline $\begin{array}{l}\text { No. of patients with SSI who required systemic } \\
\text { antibiotic treatment (\%) }\end{array}$ & $0(0)$ & $1(1.4)$ \\
\hline $\begin{array}{l}\text { No. of patients with SSI who required hospital } \\
\text { readmission (\%) }\end{array}$ & $0(0)$ & $1(1.4)$ \\
\hline $\begin{array}{l}\text { No. of patients with SSI who required surgical } \\
\text { intervention (\%) }\end{array}$ & 0.080 .030 \\
\hline \begin{tabular}{l} 
Median (range) time of SSI occurrence [days] \\
\cline { 2 - 3 }
\end{tabular} & Study group $(n=2)$ & Control group $(n=7)$ \\
\hline
\end{tabular}

Data are expressed as frequency (\%). SSI - surgical site infection.

cases of surgical wound dehiscence and none of the patients required rehospitalization or surgical intervention. Additionally, patients in whom a hydrophobic dressing was applied required systemic antibiotic therapy significantly less frequently. As a result, the use of hydrophobic dressings in standard management strategies for wounds in patients undergoing a CS may contribute to reduced total costs of treatment.

The observed incidence of wound infection after a CS, namely $2.8 \%$ or $9.8 \%$ depending on the type of dressing used, is similar to results observed by other authors [3-8]. The obtained results probably do not reflect the total SSIs rate due to the fact that the analysis was limited to superficial and deep infections without the organ/ space group, and there was a 14-day follow-up after surgery, contrary to the recommended 30day period presented in the CDC criteria [2]. Introduced changes were a result of the assumption that the rate of organ/space SSI after a CS is low, and dressings have an insignificant effect on their occurrence. The fact that within the scheduled 2 weeks of a follow-up wound infection diagnosis was performed only by a trained healthcare provider also was of importance. Our assumptions are supported by literature data reporting that superficial and deep wound infections after a CS constitute $93-100 \%$ of all SSIs, and $77-95 \%$ of them develop within the first 14 days after the surgery [4-7]. In order to provide additional verification of the results, a hospital medical database was analyzed to search for patients referred to an outpatient clinic with symptoms suggesting SSIs, and in whom the period since the surgery ranged between 15 and 30 days. There were no records corresponding to such criteria. At the same time, the study by Whitby et al. demonstrated a high negative predictive value of patient wound selfmonitoring exceeding $97 \%$, in case of lack of infection symptoms and irrespective of the patient knowledge regarding SSIs [22]. There is a slight likelihood of an error of verification performed, however, as patients might have reported to another medical centre.

According to the analysis of risk factors for surgical wound infections, only pre-pregnancy BMI was a statistically significant variable. The fact that obesity increases the risk of wound infection after a CS has been confirmed by many publications [5-8]. It is presumed that excessive thickness of the subcutaneous tissue favors hypoperfusion of tissues, resulting in their ischemia and making antibiotic therapeutic concentration difficult to achieve [23]. An additional aspect is the fact that obesity is often accompanied by carbohydrate metabolism disturbances and prolonged surgery time due to technical difficulties and the need to perform a longer skin incision [23]. It seems that due to a constantly increasing rate of obese or overweight women subject to a CS it is justified to verify and adjust prophylactic doses of antibiotics administered prior to surgery in this group of patients. Supervision by a dietician may also be of importance in the period prior to and during pregnancy.

Lack of confirmed statistical significance for other remaining risk factors for SSIs, such as diabetes, prolonged surgery time, surgeon experience or emergency mode of CS in the present study is probably a result of a too small study group compared to previous publications [5-8].

The microbiological analysis of wound cultures indicated that the main pathogen responsible for infection in both groups is coagulase-negative Staphylococcus epidermidis, strains of which exhibit high variability with regard to $\mathrm{CSH}$, and hydrophilicity is more predominant [17, 24-26]. In the group with hydrophobic dressings and in the group with a standard surgical dressing there were no cases of infection caused by $S$. aureus, whereas in the latter group the presence of Pseudomonas aeruginosa was confirmed twice. This last phenomenon can be explained by an observed increase in CSH of $P$. aeruginosa in carbon dioxide atmosphere and in the presence of serum [17]. These conditions, similar to the wound en- 
vironment under an occlusive dressing, may contribute to increased absorption of bacteria due to hydrophobic interactions.

In conclusion, it is commonly known that infection impairs and delays wound healing due to damage of healthy cells by toxins released by pathogenic microorganisms and enzymes from inflammatory cells. Only in the case of spreading or systemic infection is it recommended to use antibiotics in combination with topical antimicrobial treatment. However, when only wound contamination or colonization occurs and the healing process progresses, the use of methods as above is not recommended due to systemic action and a possibility of resistance development. Surgical wounds in obstetrics are classified as clean-contaminated wounds; therefore in infection prophylaxis it is recommended to use local methods and preferably those that limit the release of additional antimicrobial agents due to lactation. This study has demonstrated that DACC-impregnated dressings can be applied for the prevention of SSIs in women after a CS based solely on a physical mechanism of action. Taking into account the constantly increasing rate of patients subject to CS, and the fact that a considerable number of women of reproductive age have risk factors for wound infections, further attempts to use such dressings are justified and the obtained study results are promising. The study presented above was preliminary; a randomized, controlled trial with a larger group of patients is ongoing, and its aim is to draw final conclusions.

\section{Conflict of interest}

The authors declare no conflict of interest.

\section{References}

1. Betran AP, Marialdi M, Lauer JA, et al. Rates of caesarean section: analysis of global, regional and national estimates. Paediatr Perinat Epidemiol 2007; 21: 98-113.

2. Mangram AJ, Horan TC, Pearson ML, Silver LC, Jarvis WR. Guideline for prevention of surgical site infection. Infect Control Hosp Epidemiol 1999; 20: 250-78.

3. Hadar E, Melamed N, Tzadikevitch-Geffen K, Yogev Y. Timing and risk factors of maternal complications of cesarean section. Arch Gynecol Obstet 2011; 283: 735-41.

4. Wilson J, Wloch C, Saei A, et al. Inter-hospital comparison of rates of surgical site infection following caesarean section delivery: evaluation of a multicentre surveillance study. J Hosp Infect 2013; 84: 44-51.

5. Opøien HK, Valbø A, Grinde-Andersen A, Walberg M. Post-cesarean surgical site infections according to CDC standards: rates and risk factors. A prospective cohort study. Acta Obstet Gynecol 2007; 86: 1097-102.

6. Wloch C, Wilson J, Lamagni T, Harrington P, Charlett A, Sheridan E. Risk factors for surgical site infection following caesarean section in England: results from a multicentre cohort study. BJOG 2012; 119: 1324-33.
7. Olsen MA, Butler AM, Willers DM, Devkota P, Gross GA, Fraser VJ. Risk factors for surgical site infection after low transverse cesarean section. Infect Control Hosp Epidemiol 2008; 29: 477-84.

8. Schneid-Kofman N, Sheiner E, Levy A, Holcberg G. Risk factors for wound infection following cesarean deliveries. Int J Gyn Obstet 2005; 90: 10-5.

9. Jenks PJ, Laurent M, McQuarry R, Watkins R. Clinical and economic burden of surgical site infection (SSI) and predicted financial consequences of elimination of SSI from an English hospital. J Hosp Infect 2014; 86: 24-33.

10. Hampton S. An evaluation of the efficacy of Cutimed ${ }^{\circledR}$ Sorbact ${ }^{\circledR}$ in different types of non-healing wounds. Wounds UK 2007; 3: 113-9.

11. Kammerlander G, Locherer E, Süss-Burghart A, von Hallern B, Wipplinger P. Non-medicated wound dressing as an antimicrobial alternative in wound management. Wounds UK 2008; 4: 10-8.

12. Von Hallern B, Lang F. Has Cutimed ${ }^{\circledR}$ Sorbact ${ }^{\circledR}$ proved its practical value as an antimicrobial dressing. Medizin und Praxis Spezial 2005; 2-7.

13. Mussi C, Salvioli G. Clinical evaluation of Sorbact (bacteria absorbing dressing) in the treatmnt of infected pressure sores. Acta Vulnol 2004; 2: 9-11.

14. Doyle RJ. Contribution of the hydrophobic effect to microbial adhesion. Microbes Infect 2000; 2: 391-400.

15. Ljungh A, Hjerten S, Wadström T. High surface hydrophobicity of aggregating Staphylococcus aureus strains isolated form human infections studied with the salt aggregation test, SAT. Infect Immun 1985; 47: 522-6.

16. Ljungh A, Österlind $M$, Wadström T. Cell surface hydrophobicity of group D and viridans streptococci isolated from patients with septicemia. Zbl Bakteriol Mikrobiol Hyg A 1986; 261: 280-6.

17. Ljungh A, Wadström T. Growth conditions influence expression of cell surface hydrophobicity of staphylococci and other wound infection pathogens. Microbiol Immunol 1995; 39: 753-7.

18. Ljungh A, Yanagisawa N, Wadström T. Using the principle of hydrophobic interaction to bind and remove wound bacteria. J Wound Care 2006; 15: 175-80.

19. Gentili S, Gianesini P, Balboni E, Menegatti A, Rotola M. Panbacterial real-time PCR to evaluate bacterial burden in chronic wounds treated with CutimedTM SorbactTM. Eur J Clin Microbiol Infect Dis 2012; 31: 1523-9.

20. Falk P, Ivarsson ML. Effect of a DACC dressing on the growth properties and proliferation rate of cultured fibroblasts. J Wound Care 2012; 21: 327-32.

21. Schulz KF, Altman DG, Moher D; for the CONSORT Group. CONSORT 2010 Statement: updated guidelines for reporting parallel group randomised trials. BMJ 2010; 340: c332.

22. Whitby M, McLaws ML, Doidge S, Collopy B. Post-discharge surgical site surveillance: does patient education improve reliability of diagnosis? J Hosp Infect 2007; 66: 237-42

23. Anaya DA, Dellinger E. The obese surgical patient: a susceptible host for infection. Surg Infect 2006; 7: 473-80.

24. Molnar C, Hevessy Z, Rozgonyi F, Gemmell CG. Pathogenicity and virulence of coagulase negative staphylococci in relation to adherence, hydrophobicity, and toxin production in vitro. J Clin Pathol 1994; 47: 743-8.

25. Kristinsson KG. Adherence of staphylococci to intravascular catheters. J Med Microbiol 1989; 28: 249-57.

26. Hogt AH, Dankert J, Feijen J. Encapsulation, slime production and surface hydrophobicity of coagulase-negative staphylococci. FEMS Microbiol Lett 1983; 18: 211-5. 